\title{
Biatrial versus Isolated Left Atrial Ablation in Atrial Fibrillation: A Systematic Review and Meta-Analysis
}

\author{
Hongmu Li, ${ }^{1}$ Xifeng Lin, ${ }^{2}$ Xun Ma, ${ }^{3}$ Jun Tao, ${ }^{2}$ Rongjun Zou, ${ }^{2}$ Songran Yang $\left(\mathbb{D},{ }^{4,5}\right.$ \\ Haibo Liu $\left(\mathbb{1},{ }^{1}\right.$ and Ping Hua $\mathbb{\circledR}^{2}$ \\ ${ }^{1}$ Department of Cardiovascular Surgery, Quanzhou First Hospital of Fujian Medical University, Quanzhou 362000, China \\ ${ }^{2}$ Department of Cardiovascular Surgery, Sun Yat-sen Memorial Hospital, Sun Yat-sen University, Guangzhou 510120, China \\ ${ }^{3}$ Guangzhou Women and Children's Medical Center, Guangzhou 510623, China \\ ${ }^{4}$ The Biobank of Sun Yat-sen Memorial Hospital, Sun Yat-sen University, Guangzhou 510120, China \\ ${ }^{5}$ Guangdong Province Key Laboratory of Brain Function and Disease, Zhongshan School of Medicine, \\ Sun Yat-sen University, Guangzhou 510080, China
}

Correspondence should be addressed to Songran Yang; yangsongran@126.com, Haibo Liu; haibo_liu01@163.com, and Ping Hua; huaping88@sina.com

Received 3 October 2017; Revised 10 March 2018; Accepted 14 March 2018; Published 29 April 2018

Academic Editor: Francesco Onorati

Copyright (C) 2018 Hongmu Li et al. This is an open access article distributed under the Creative Commons Attribution License, which permits unrestricted use, distribution, and reproduction in any medium, provided the original work is properly cited.

\begin{abstract}
Objective. The outcomes of biatrial ablation (BA) and isolated left atrial ablation (LA) in atrial fibrillation remain inconclusive. In this meta-analysis, we assess the currently available evidence to compare outcomes between BA and LA. Methods. Electronic searches were performed from database inception to December 2016, and relevant studies were accessed. Odds ratios and weight mean differences with 95\% confidence intervals are reported. Twenty-one studies comprising 3609 patients were included in the present meta-analysis. Results. The prevalence of sinus rhythm in the BA cohort was similar to that in the LA cohort at discharge, at 12 months, and after more than 1 year of follow-up. However, at 6 months, the prevalence of sinus rhythm was higher in the BA cohort than in the LA cohort. The rate of permanent pacemaker implantation was higher in the BA cohort than in the LA cohort. However, 30-day and late mortality and neurological events were similar between the BA and LA groups. Conclusion. There was no significant difference in the rate of restored sinus rhythm, the risk of death, and cerebrovascular events between BA and LA, but BA had a higher rate of permanent pacemaker implantation.
\end{abstract}

\section{Introduction}

Atrial fibrillation (AF) is a major healthcare problem worldwide which has enormous economic and public health implications. AF is associated with an increased risk of stroke, heart failure, and all-cause mortality [1-3].

Surgical ablation was introduced as a treatment option by Cox et al. [4] in 1991, and it is currently an effective curative strategy for AF. Haïssaguerre et al. [5] suggested that ectopic beats from pulmonary veins may cause $\mathrm{AF}$, and the field of pulmonary vein isolation (PVI) was consequently established and is now performed via catheter or surgical ablation [6]. Left ablation (LA) has historically been the main method used to treat AF, and it has a fairly good clinical effect $[7,8]$. However, some studies have suggested that LA is less efficacious than biatrial ablation (BA), especially when a right-side AF trigger is present [9]. Hence, because outcomes have been inconclusive, Phan et al. [10] and Zheng et al. [11] reported relevant meta-analyses in 2014, but they arrived at a different conclusion. The authors showed that BA was more effective than LA and that the rate of permanent pacemaker implantation was also higher in the BA cohort than in the LA cohort. However, Zheng et al. [11] suggested that the effects of $\mathrm{BA}$ and LA are the same.

In the past two years, several other studies $[8,12-15]$ have compared BA and LA in AF, with controversial outcomes. Hence, in this meta-analysis, we sought to assess the current evidence available on this issue. 


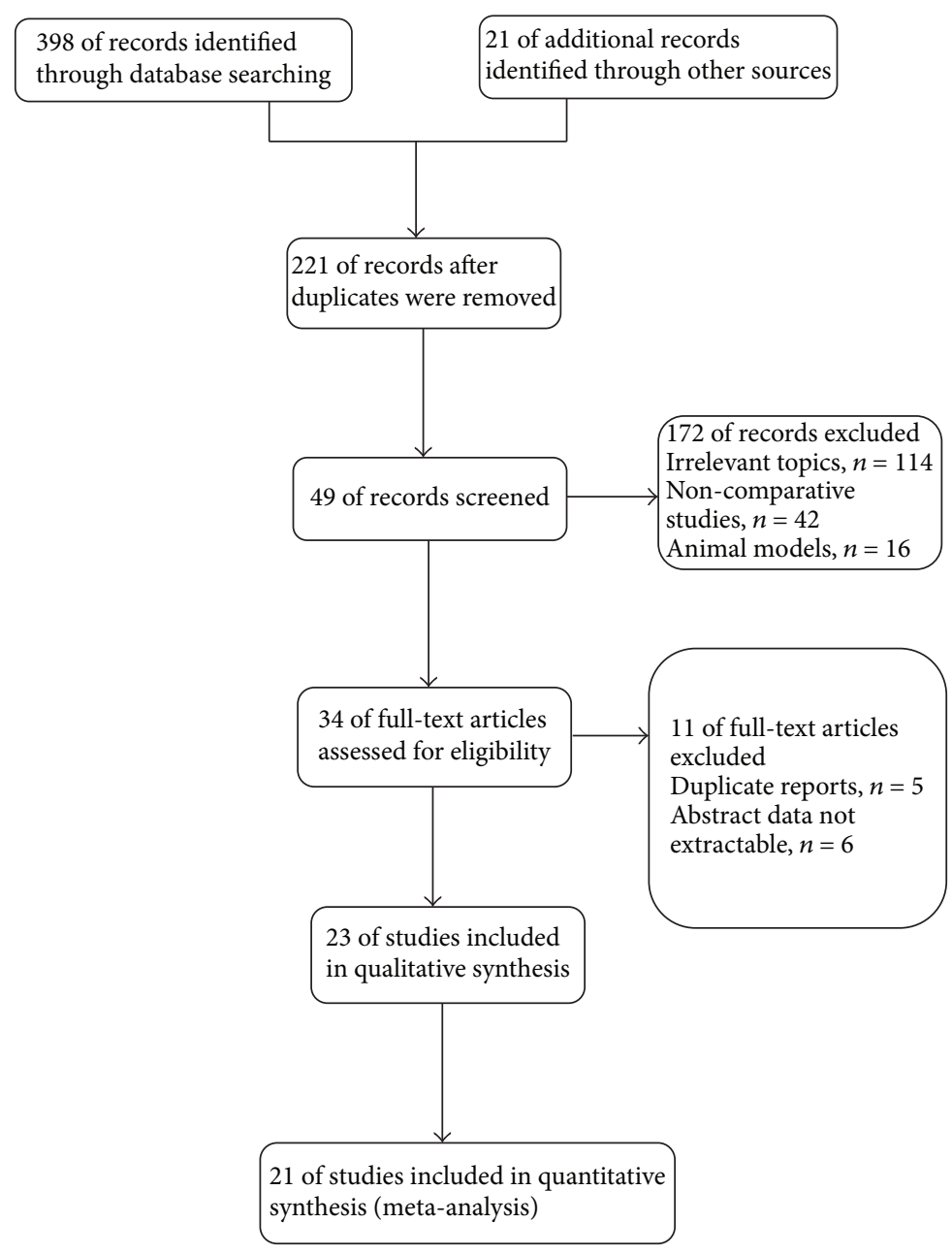

FIGURE 1: Selection of studies for the meta-analysis.

\section{Methods}

2.1. Literature Search Strategy. Electronic searches were performed in August 2016 without search restrictions. The primary sources were the electronic Medline, PubMed, Cochrane Library, and EMBASE databases, which were searched from their date of inception to August 2016. The following search terms were used: "maze," "biatrial," "biatrial," "uniatrial," "left atrial," and "ablation.” When duplicate published trials with accumulating numbers of patients or increased lengths of follow-up were encountered, the most recent or most complete report was considered. All titles and abstracts identified in the electronic search were uploaded into an EndNote (version X7; Thomson Corporation, Stanford, USA) database (Figure 1).

2.2. Inclusion and Exclusion Criteria. All available randomized, controlled trials (RCTs) and retrospective comparative studies that compared BA with LA in all age groups were included. Abstracts, case reports, conference presentations, editorials, reviews, and expert opinions were excluded. When institutions published duplicate studies with accumulating numbers of patients or increased lengths of follow-up, only the most complete report was included. Reference lists were also manually searched for further relevant studies.

2.3. Data Extraction and Critical Appraisal. Two reviewers (Hongmu Li and Xifeng Lin) conducted data extraction independent of the included studies. Data on authorship, year of publication, study design, study population, baseline characteristics, characteristics related to outcomes, and duration of follow-up were extracted from each study. Reported percentages were approximated to numbers. The risk of bias was assessed using the Downs and Black checklist [16] for randomized and observational studies. Discrepancies between the reviewers were resolved by discussion until consensus was reached. The final results were reviewed by the senior investigator (Ping Hua).

2.4. Quality Assessment and Statistical Analysis. The included studies were rated to determine the level of quality of the provided evidence according to the criteria of the Centre for Evidence-Based Medicine in Oxford, UK [17]. The methodological quality of the RCTs was assessed with the 


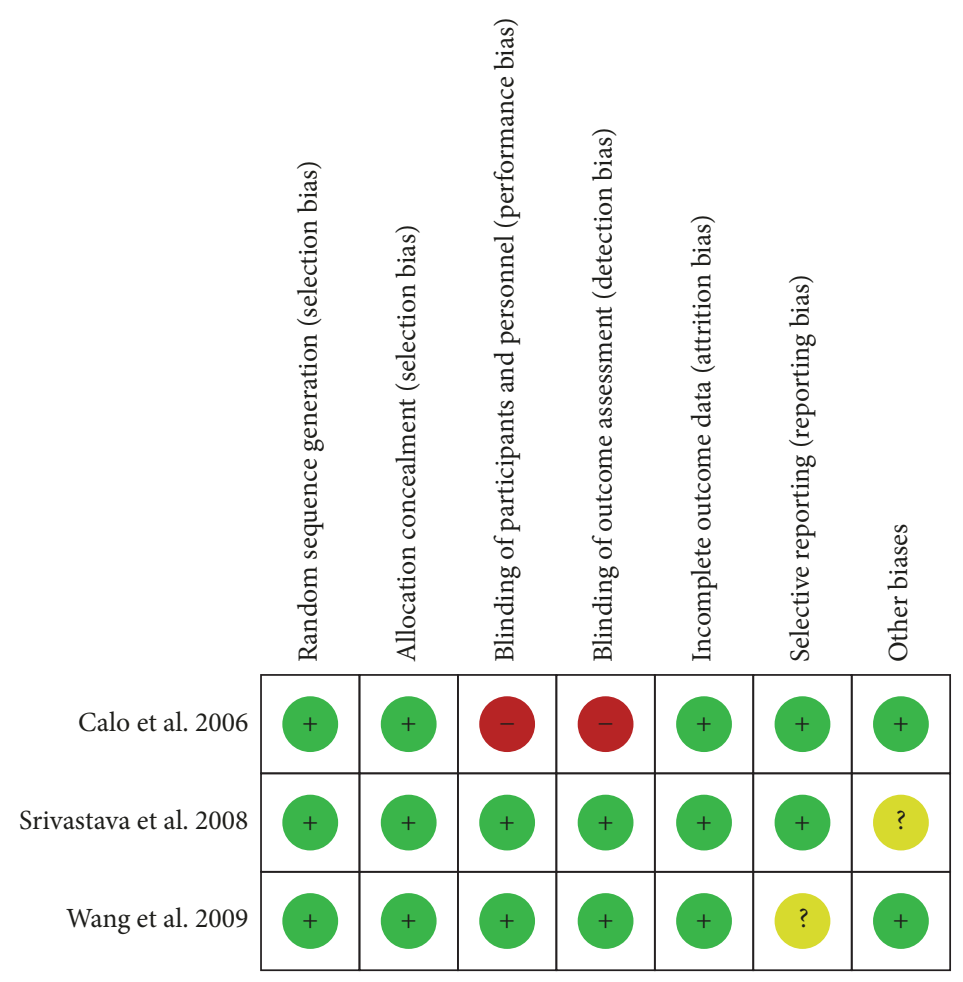

FIgURE 2: Risk of bias in RCT studies.

Cochrane risk of bias tool [18]. The methodological quality of retrospective studies was assessed with the modified Newcastle-Ottawa scale [19, 20].

This meta-analysis was performed using Review Manager Version 5.3 (Cochrane Collaboration, Oxford, UK). Dichotomous variables from individual studies were analyzed using odds ratios (ORs) with 95\% confidence intervals (CIs). Qstatistics $(P<0.10)$ or $I^{2}$ statistics were performed to test for heterogeneity between included studies, and values of $50 \%$ or higher were considered to be indicative of substantial heterogeneity. If there was substantial heterogeneity, the possible clinical and methodological reasons for this were explored qualitatively. Publication bias was examined through a visual inspection of funnel plots and assessed by applying Egger's weighted regression statistic and considering a $P$ value less than 0.05 as indicating significant publication bias. A $P$ value of less than 0.05 was considered statistically significant for all analyses.

2.5. Synthesis of Evidence. Our electronic literature search resulted in the retrieval of 398 citations. Of these, 372 were excluded after duplicate and irrelevant references were excluded, and 49 potentially relevant articles were retrieved. Finally, following a manual search of reference lists and a critical appraisal, 21 studies comprising 3609 patients were included in this meta-analysis. Two articles had redundant publications but covered different characteristics [21, 22].

2.6. Quality Assessment and Baseline Characteristics of Eligible Studies. In all, 21 studies were included in this meta-analysis, including three prospective randomized trials [23-25], five prospective observational studies [13, 26-29], and 13 retrospective observational studies $[8,12,14,15,21,22,30-36]$. The risk of bias in each study is shown in Figure 2 and Table 1. Among the 3609 patients, 1901 received BA, and 1708 received LA. Patients in the BA group underwent a classical or modified maze procedure, including both leftsided and right-sided maze procedures. However, patients in the LA group underwent a left-sided maze procedure that included PVI, left atrial posterior wall isolation, mitral isthmus ablation, and left atrial appendage excision.

Three studies used cryoablation energy $[15,27,31]$, eleven studies used radiofrequency energy [12-14, 21, 23, 25, 26, 29, 32-34], and the remaining studies used a combination of different energy sources, including radiofrequency, cryoablation, and microwave and "cut-and-sew" [8, 22, 24, 28, 30, $35,36]$. Concomitant coronary artery bypass grafting surgery was reported in 10 studies [8, 13, 21, 22, 25, 27-31, 36], while a concomitant valvular operation was performed in other included studies. The baseline characteristics of the patients in the included studies are shown in Tables 2 and 3.

\section{Outcomes}

3.1. Assessment of Efficacy. The data were pooled from 16 studies $[12-15,21-26,28,29,31-33,36]$ that assessed the efficacy of restoring sinus rhythm (SR), and the results showed that there was no significant difference between the BA and LA groups at discharge (78.3\% versus $73.86 \%$; OR: 1.02; 95\% CI: $0.69-1.51 ; P=0.92 ; I^{2}=66 \%$ ). However, the overall prevalence of SR was higher in the BA group than in the LA group at a 6-month follow-up (78.82\% versus 


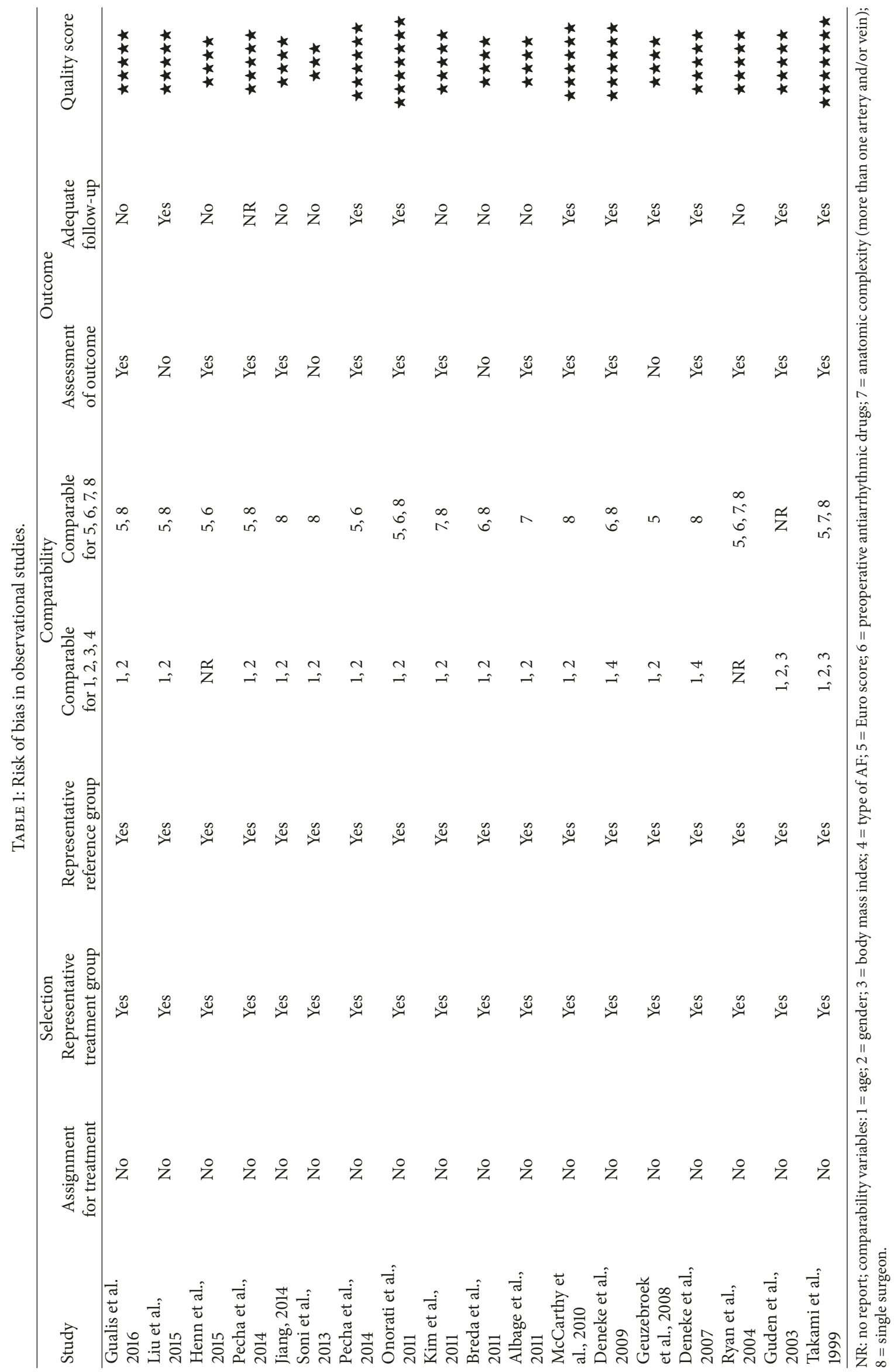




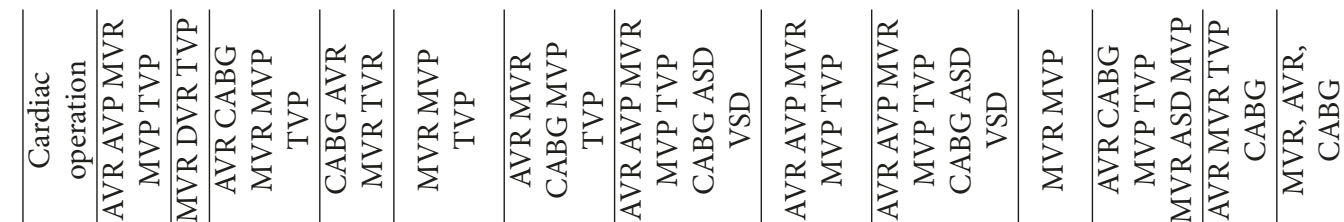

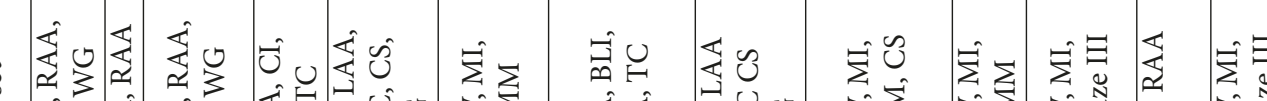

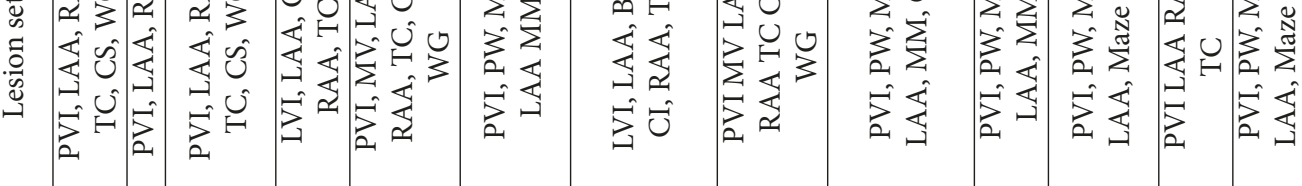

.

$\ddot{\circ}$

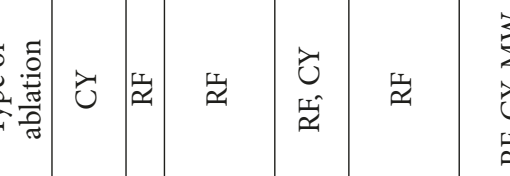

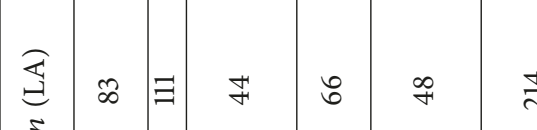

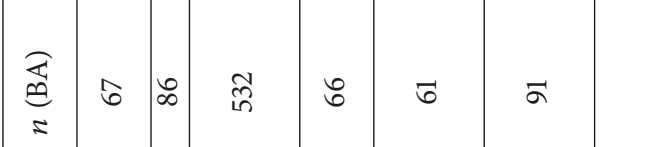



ồ

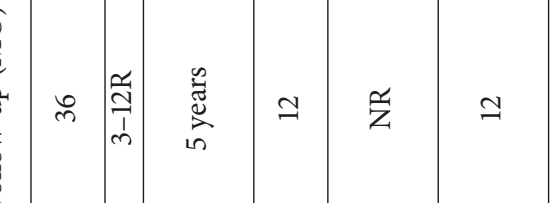

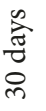

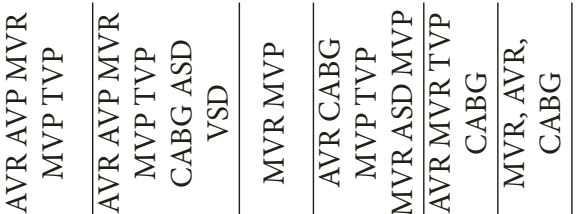

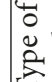

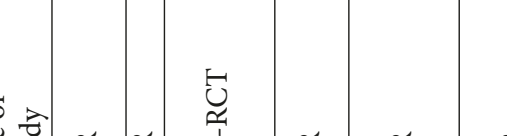

$\simeq$

$\vec{m}$

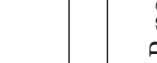

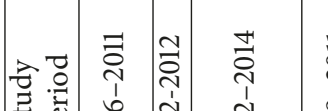

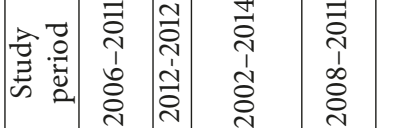

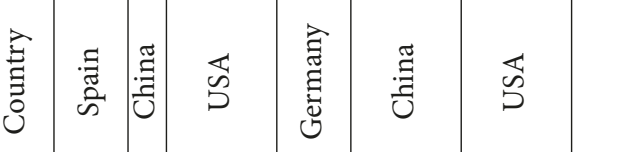

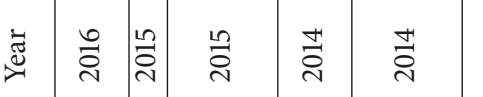

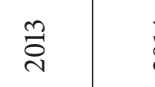

突

न

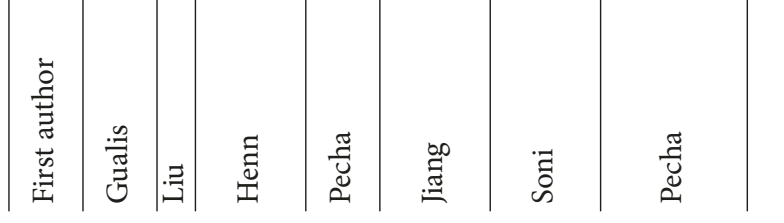

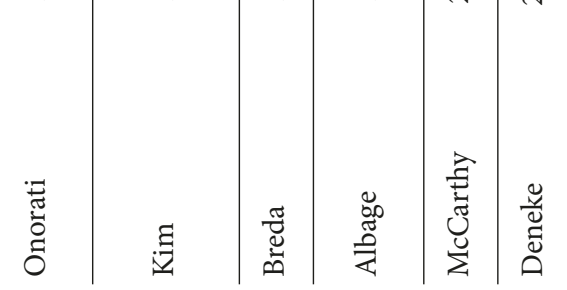




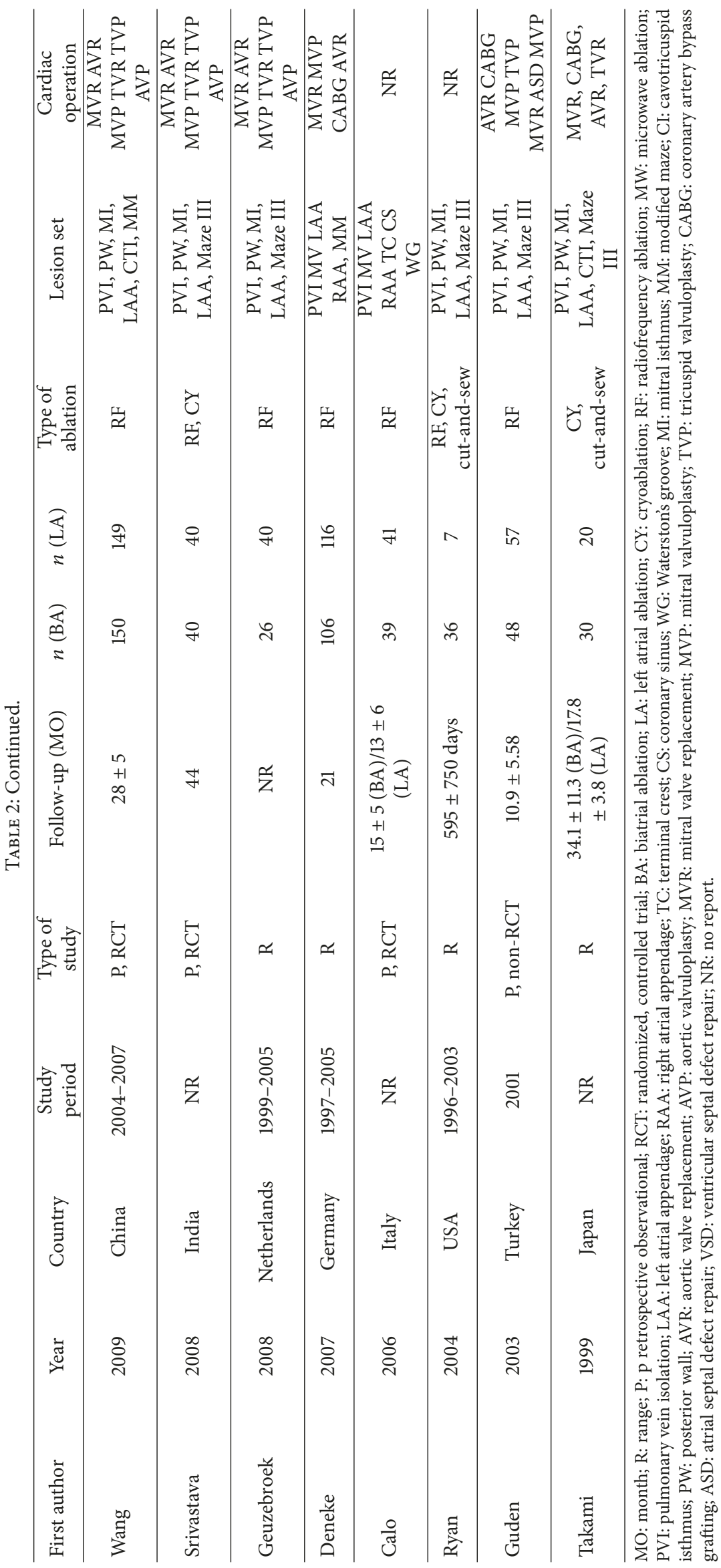




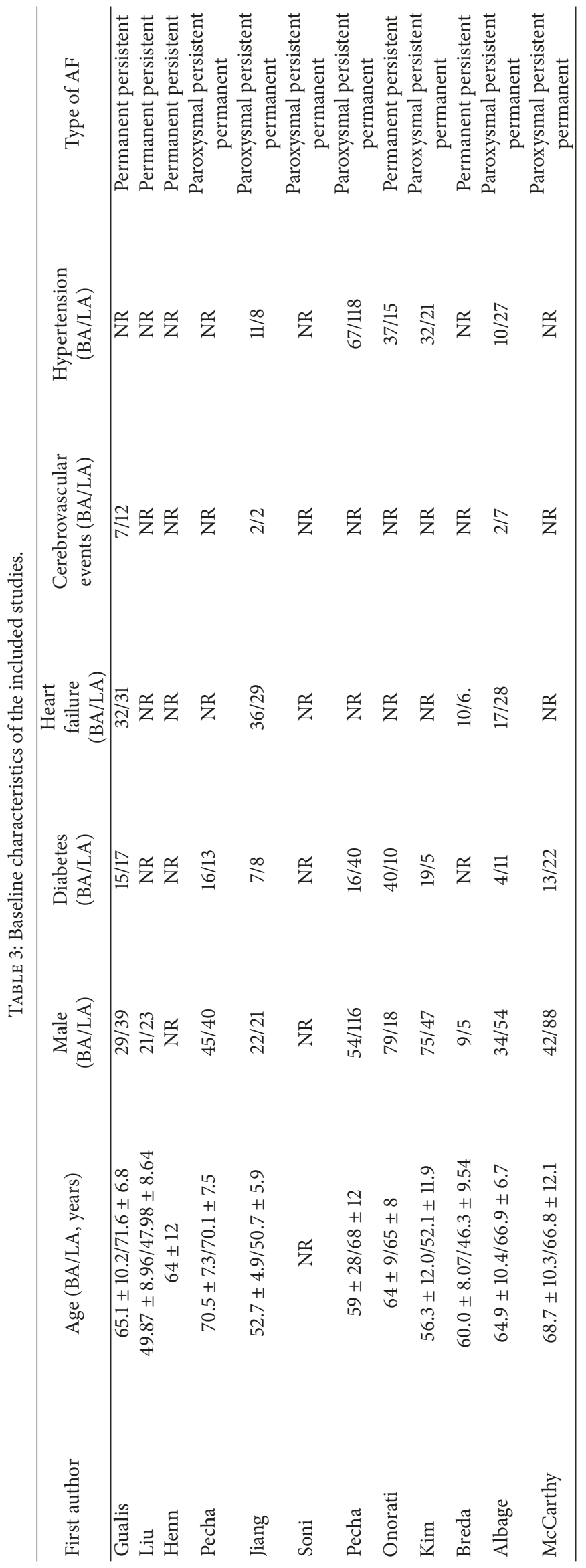




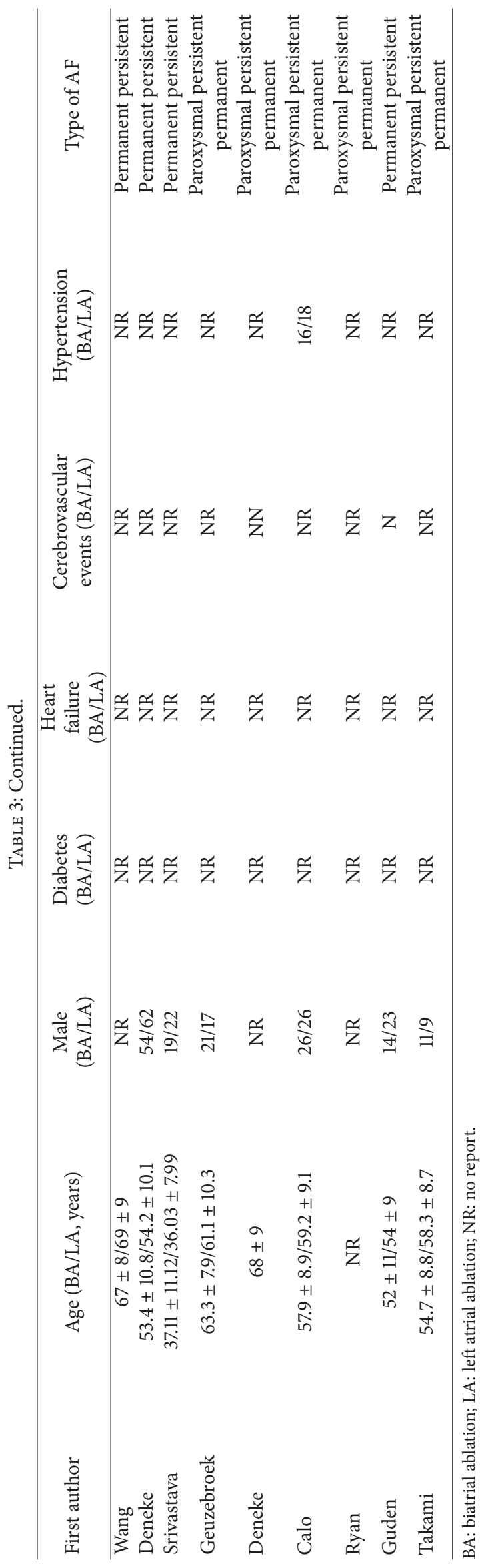


TABLE 4: Sensitivity and subgroup analyses.

\begin{tabular}{|c|c|c|c|c|c|c|}
\hline \multirow{2}{*}{ Endpoint } & \multicolumn{2}{|c|}{ Restored SR at discharge } & \multicolumn{2}{|c|}{ Restored SR at 12 months } & \multicolumn{2}{|c|}{ Restored SR beyond 1 year } \\
\hline & Overall $P$ value & OR $(95 \% \mathrm{CI})$ & Overall $P$ value & OR $(95 \% \mathrm{CI})$ & Overall $P$ value & OR $(95 \% \mathrm{CI})$ \\
\hline Study design & & & & & 0.46 & $1.37(0.60,3.11)$ \\
\hline RCT & 0.38 & $0.51(0.12,2.25)$ & \multirow{2}{*}{\multicolumn{2}{|c|}{ Have no RCT studies }} & 0.69 & $1.25(0.43,3.65)$ \\
\hline Non-RCT & 0.4 & $1.17(0.81,1.68)$ & & & & \\
\hline \multicolumn{7}{|l|}{ Study size } \\
\hline$<150$ & 0.88 & $1.05(0.56,1.95)$ & 0.04 & $1.76(1.02,3.04)$ & 0.76 & $1.22(0.24,4.46)$ \\
\hline$>150$ & 0.96 & $0.99(0.66,1.49)$ & 0.44 & $1.29(0.67,2.48)$ & 0.3 & $1.44(0.73,2.85)$ \\
\hline \multicolumn{7}{|l|}{ Statistical models } \\
\hline Fixed-effect & 0.3 & $1.12(0.90,1.38)$ & 0.009 & $1.39(1.09,1.79)$ & 0.16 & $1.21(0.92,1.59)$ \\
\hline Random-effect & 0.34 & $1.15(0.86,1.55)$ & 0.2 & $1.37(0.85,2.21)$ & 0.4 & $1.31(0.70,2.48)$ \\
\hline
\end{tabular}

SR: sinus rhythm; RCT: randomized, controlled trial.

69.67\%; OR: $1.54 ; 95 \%$ CI: $\left.1.17-2.03 ; P=0.002 ; I^{2}=0 \%\right)$ $[14,15,22,24-26,30-32,36]$. For patients with a follow-up at 12 months $[8,14,15,26,27,30,31,34]$ and after more than 1 year $[12,15,23-25,31]$, the prevalence of SR in the BA group was similar to that in the LA group (63.01\% versus 65.47\%; OR: 1.31; 95\% CI: 0.70-2.48; $\left.P=0.40 ; I^{2}=77 \%\right)$. The weighted average mean follow-up for studies reporting SR after more than 1 year was 23.3 months. These results are shown in Figure 3.

3.2. Mortality and Major Complications. Eight studies with 1185 patients investigated mortality after the BA or LA procedure. When effects were pooled, there was no significant difference in either early mortality $[8,14,23,27-29,33](<30$ days, OR: 1.02; 95\% CI: $0.36-2.90 ; P=0.97 ; I^{2}=31 \%$, Figure 4) or late mortality [23, 27, 29, 31, 35] (OR: 2.31; 95\% CI: $0.86-6.22 ; P=0.10 ; I^{2}=0 \%$, Figure 5) between the BA and LA groups.

There was no significant increase in the risk of cerebrovascular events $[8,27,28,31]$ between the two groups (OR: 0.61; 95\% CI: $0.16-2.40 ; P=0.48 ; I^{2}=0 \%$, Figure 6). In eight studies that compared LA with BA, BA increased the risk of permanent pacemaker implantation $[22,23,30,31,33,36]$ (OR: 2.46 ; 95\% CI: $1.55-3.91 ; P=0.0001 ; I^{2}=0 \%$, Figure 7 ). No heterogeneity was observed.

3.3. Sensitivity Analysis and Publication Bias. The risk of bias was comprehensively assessed according to the guidelines of the Cochrane Collaboration, and neither visual inspection of funnel plots nor Egger's test detected significant publication bias for the major outcomes explored in this meta-analysis, including the prevalence of SR at discharge $(t=0.04 ; P=$ $0.972), \mathrm{SR}$ at a 6-month follow-up $(t=0.27 ; P=0.791)$, SR at a 12-month follow-up $(t=0.90 ; P=0.401)$, SR after more than 1 year $(t=0.52 ; P=0.626)$, early mortality $(t=1.03 ; P=0.363)$, late mortality $(t=-1.07 ; P=0.397)$, neurological events $(t=51.13 ; P=0.012)$, and permanent pacemaker implantation $(t=2.42 ; P=0.060)$. To evaluate the effect of heterogeneity on the pooled effect, we carried out a sensitivity analysis. Sensitivity and subgroup analyses found no significant heterogeneity (Table 4 ).

\section{Discussion}

Two recent meta-analyses $[10,11]$ that compared BA, LA, and surgical ablation in AF arrived at conflicting conclusions. However, these meta-analyses excluded several studies that compared BA with LA $[8,12-15,25]$. Therefore, we performed a new meta-analysis to compare BA with LA. This metaanalysis included three RCTs and 18 retrospective studies that collectively contained 3609 patients and compared the efficacy and safety of BA and LA. There was no significant difference between BA and LA in the rate of restored SR, but BA groups had a higher probability of SR after 6 months of follow-up. We also found that while BA and LA had similar rates of death and cerebrovascular events, the BA groups had a higher rate of permanent pacemaker implantation.

A pooled analysis of restored postoperative SR showed that there was no difference between the BA and LA groups. However, several recent studies $[21,23,36]$ have shown that $\mathrm{BA}$ is inferior to the more complete LA when used alone. Patients in the LA group had shorter aortic cross-clamping times and cardiopulmonary bypass times than were observed in the BA group. Furthermore, the techniques used in AF ablation vary widely, even within the same procedure group, and if the different lesion sets used for ablation were included, the results may have indicated that this procedure has greater efficacy.

In contrast, some studies have reported that $\mathrm{BA}$ is superior to LA for restoring SR [8, 12, 15, 25-28, 30-33]. This finding rests mainly on the finding that BA groups have much more tissue damage and a higher rate of cardiac conduction system injury. However, all of these studies have common limitations. First, some patients took antiarrhythmic drugs (including amiodarone) perioperatively and continued the use of these drugs until the operation, and few researchers sufficiently addressed this variable. Second, the sample size in most of the articles was small (less than 150 individuals), weakening the power of the studies. Third, long rhythm registration during follow-up was not available in all of the patients. Furthermore, only a few of the studies were RCTs. Unlike previous reviews, we included the largest studies in our meta-analysis, and our inclusion criteria did not limit our search to articles published in English. We also conducted 


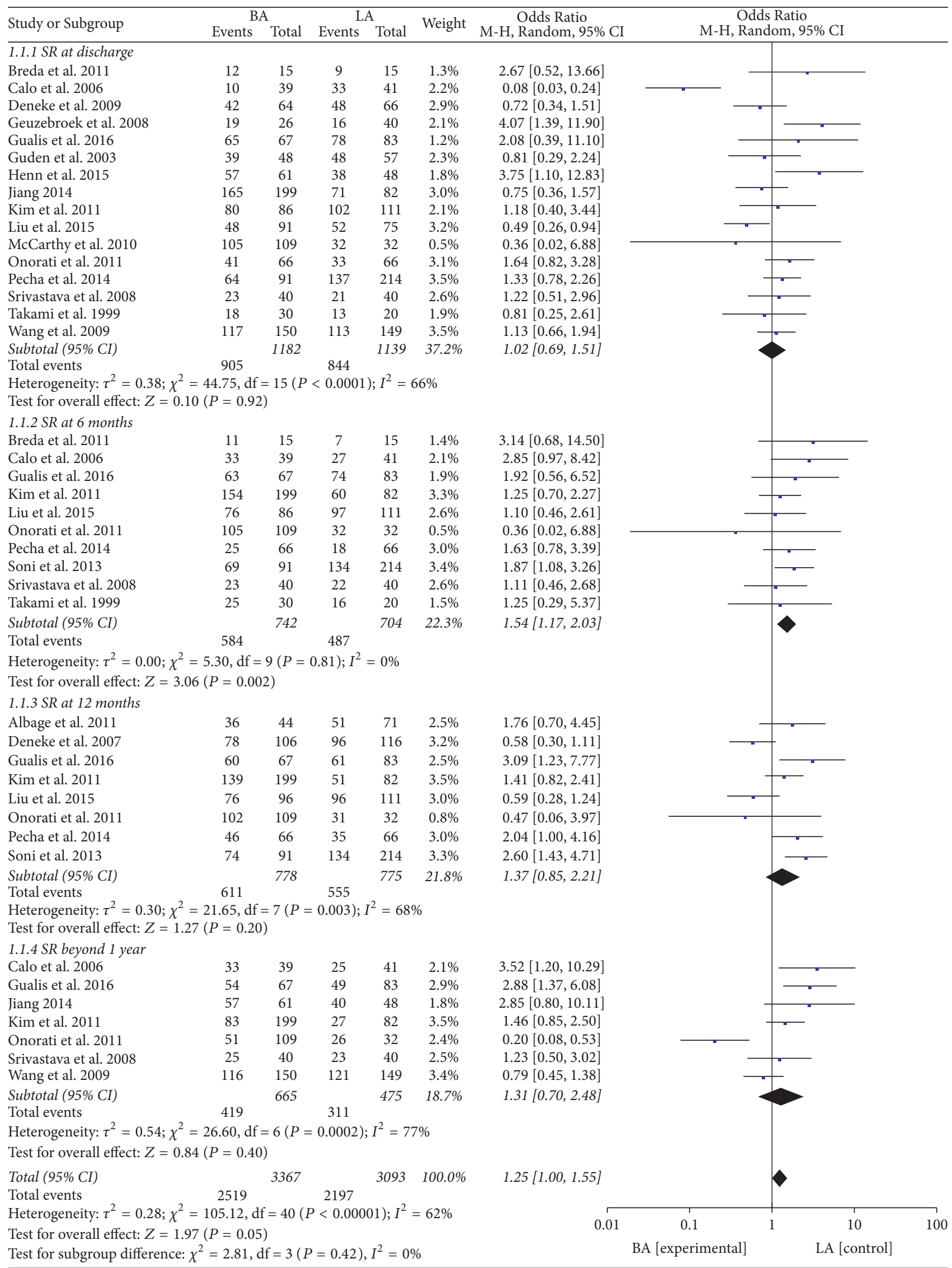




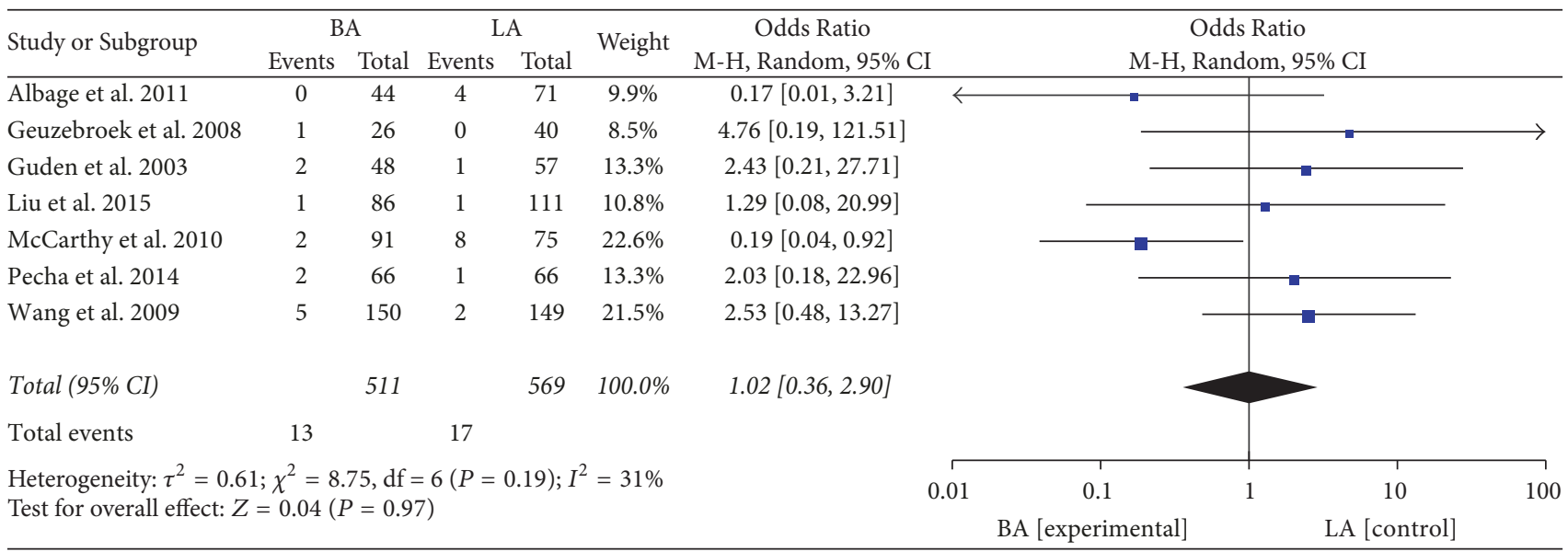

FIGURE 4: Mortality within 30 days.

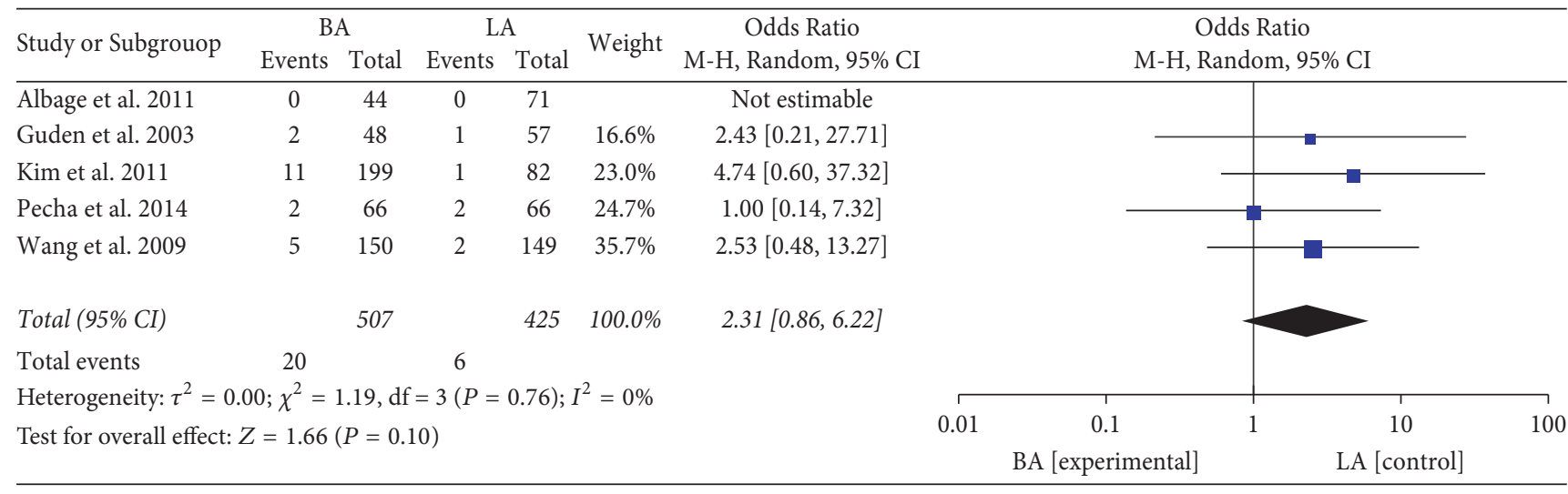

FIGURE 5: Late mortality.

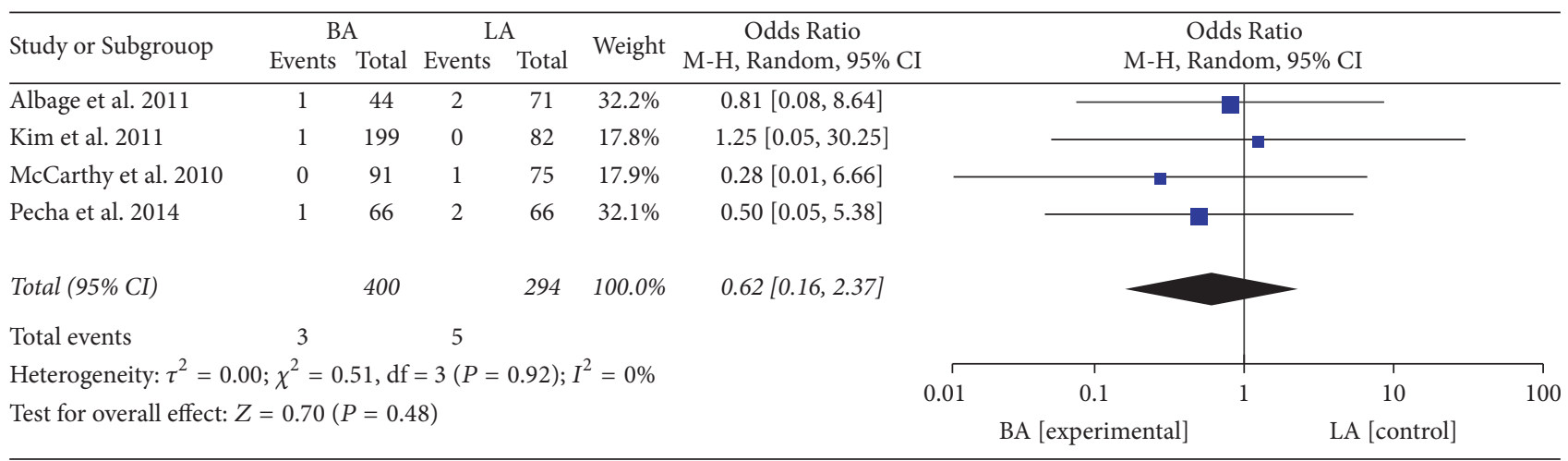

FIGURE 6: Cerebrovascular events.

a subgroup analysis of RCT and non-RCT studies and of small-sample and large-sample studies to assess the effect of heterogeneity on the pooled effect estimate.

The findings of the present meta-analysis confirm that $\mathrm{BA}$ increases the risk of permanent pacemaker implantation. This finding may be attributed to the fact that LA has shorter aortic cross-clamping and cardiopulmonary bypass times and promotes more extensive lesions. There was no significant increase in the risk of cerebrovascular events or early and late mortality between the two groups. We hypothesize that report selection resulted in fewer such events, and these results remain to be discussed.

The most important findings of our meta-analysis include the following: (1) LA and BA were equally effective in restoring SR, (2) BA resulted in higher prevalence of SR at the 6-month follow-up, and (3) unlike previous analyses, this 


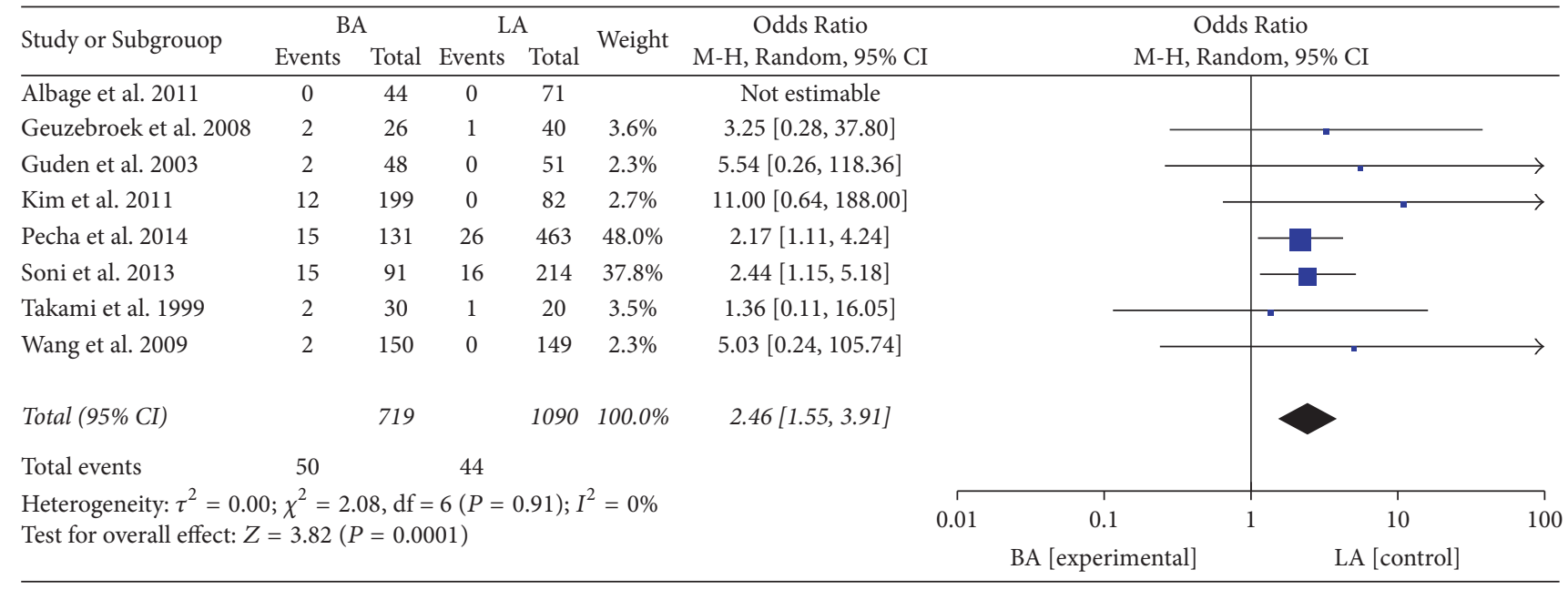

FIgURe 7: Permanent pacemaker implantation.

meta-analysis included the largest studies, and its inclusion criteria did not limit the search to articles published in the English language.

The results of our study show that there was no difference in the rate of restored SR between LA and BA. While some previous studies have proposed that $\mathrm{BA}$ alone is inferior to a more complete LA, this significance disappeared in a multivariate analysis. The difference in these results may have been caused by differences in inclusion criteria between previous studies and our study. The other studies limited inclusion to articles reported in the English language. Additionally, the techniques used for AF ablation varied widely, including, for example, the use of different lesion sets, even within same procedure group.

One of the most important reasons that researchers have suggested for why BA is better than LA at restoring SR is that there is a significant difference in electrical activity between patients with chronic and paroxysmal AF [37, 38]. Lazar et al. [37] demonstrated that a left-to-right atrial frequency gradient exists in paroxysmal but not persistent AF. This prompted them to propose that the maintenance of persistent or chronic AF may be less dependent on the posterior left atrium. Additionally, Sanders et al. [39] proposed that, in patients with paroxysmal AF, the dominant sources of activity are often localized in the pulmonary veins. In contrast, in patients with permanent AF, the dominant sites are more often localized in the atria, including right atrial sites. Unsurprisingly, patients with persistent or long-standing persistent $\mathrm{AF}$ are more likely to receive $\mathrm{BA}$, and this may affect clinical outcomes [25].

The present meta-analysis has the following limitations. Its main limitation is that only three small-sample RCTs were included. Inadequate random sequence generation and blinding tend to increase the risk of bias. Hence, larger RCTs are needed to determine the best treatment. Another limitation is that the original meta-analysis was based on the assumption that the surgical subgroups (BA and LA) were sufficiently similar to be assessed together, but the operation methods and ablation technologies used in these procedures are continually developing. Additionally, there was extreme heterogeneity among the studies in study design, data, and energy source, and a subgroup analysis yielded results that differed from those obtained in the original analysis. Future systematic reviews should, when sufficient literature is available, evaluate different indications separately. Finally, follow-up periods were generally short. Therefore, the long-term outcomes of BA and LA remain to be explored.

\section{Conclusion}

In this comparative meta-analysis, we show that BA is not more efficacious than LA in restoring SR. Additionally, the risks of death and cerebrovascular events are significantly different between BA and LA, but BA results in a higher rate of permanent pacemaker implantation.

\section{Conflicts of Interest}

The authors declare that they have no conflicts of interest.

\section{Authors' Contributions}

Hongmu Li and Xifeng Lin contributed equally to this article.

\section{Acknowledgments}

This work was supported by the National Natural Science Foundation of China (NSFC) (no. 81771165), the Natural Science Foundation Project in Guangdong province, China (Grant no. 2016A030313295), the Major Project Development and Emerging, Interdisciplinary Funding Projects of Sun Yat-sen University (Grant no. 15ykjc17b), Guangzhou Science and Technology Project of Major Special Research Topics on International Collaborative Innovation (Grant no. 201704030032), and the grants from the Health System Training Project in Fujian Province (2015ZQNJC38). 


\section{References}

[1] S. S. Chugh, R. Havmoeller, K. Narayanan et al., "Worldwide epidemiology of atrial fibrillation: a global burden of disease 2010 study," Circulation, vol. 129, no. 8, pp. 837-847, 2014.

[2] V. Fuster, L. E. Rydén, D. S. Cannom et al., “2011 ACCF/AHA/HRS focused updates incorporated into the ACC/ AHA/ESC 2006 Guidelines for the management of patients with atrial fibrillation: a report of the American College of Cardiology Foundation/American Heart Association Task Force on Practice Guidelines developed in partnership with the European Society of Cardiology and in collaboration with the European Heart Rhythm Association and the Heart Rhythm Society," Journal of the American College of Cardiology, vol. 57, no. 11, pp. e101-e198, 2011.

[3] A. Sheikh, N. J. Patel, N. Nalluri et al., "Trends in hospitalization for atrial fibrillation: epidemiology, cost, and implications for the future," Progress in Cardiovascular Diseases, vol. 58, no. 2, pp. 105-116, 2015.

[4] J. L. Cox, T. E. Canavan, R. B. Schuessler et al., "The surgical treatment of atrial fibrillation. II. Intraoperative electrophysiologic mapping and description of the electrophysiologic basis of atrial flutter and atrial fibrillation," The Journal of Thoracic and Cardiovascular Surgery, vol. 101, pp. 406-426, 1991.

[5] M. Haïssaguerre, P. Jaïs, D. C. Shah et al., "Spontaneous initiation of atrial fibrillation by ectopic beats originating in the pulmonary veins," The New England Journal of Medicine, vol. 339, no. 10, pp. 659-666, 1998.

[6] H. Calkins, K. H. Kuck, R. Cappato et al., "2012 HRS/EHRA/ ECAS expert consensus statement on catheter and surgical ablation of atrial fibrillation: recommendations for patient selection, procedural techniques, patient management and follow-up, definitions, endpoints, and research trial design: a report of the Heart Rhythm Society (HRS) Task Force on Catheter and Surgical Ablation of Atrial Fibrillation. Developed in partnership with the European Heart Rhythm Association (EHRA), a registered branch of the European Society of Cardiology (ESC) and the European Cardiac Arrhythmia Society (ECAS); and in collaboration with the American College of Cardiology (ACC), American Heart Association (AHA), the Asia Pacific Heart Rhythm Society (APHRS), and the Society of Thoracic Surgeons (STS). Endorsed by the governing bodies of the American College of Cardiology Foundation, the American Heart Association, the European Cardiac Arrhythmia Society, the European Heart Rhythm Association, the Society of Thoracic Surgeons, the Asia Pacific Heart Rhythm Society, and the Heart Rhythm Society," Heart Rhythm, vol. 9, pp. 632-696.e621, 2012.

[7] M. Guden, B. Akpinar, I. Sanisoglu, E. Sagbas, and O. Bayindir, "Intraoperative saline-irrigated radiofrequency modified Maze procedure for atrial fibrillation," The Annals of Thoracic Surgery, vol. 74, no. 4, pp. S1301-S1306, 2002.

[8] S. Pecha, T. Schäfer, Y. Yildirim et al., "Predictors for permanent pacemaker implantation after concomitant surgical ablation for atrial fibrillation," The Journal of Thoracic and Cardiovascular Surgery, vol. 147, no. 3, pp. 984-988, 2014.

[9] F. Onorati, A. Esposito, G. Messina, A. di Virgilio, and A. Renzulli, "Right isthmus ablation reduces supraventricular arrhythmias after surgery for chronic atrial fibrillation," The Annals of Thoracic Surgery, vol. 85, no. 1, pp. 39-48, 2008.

[10] K. Phan, A. Xie, Y.-C. Tsai, N. Kumar, M. La Meir, and T. D. Yan, "Biatrial ablation vs. left atrial concomitant surgical ablation for treatment of atrial fibrillation: A meta-analysis," Europace, vol. 17, no. 1, pp. 38-47, 2014.
[11] S. Zheng, H. Zhang, Y. Li, J. Han, Y. Jia, and X. Meng, "Comparison of left atrial and biatrial maze procedure in the treatment of atrial fibrillation: a meta-analysis of clinical studies," The Thoracic and Cardiovascular Surgeon, vol. 64, no. 8, Article ID 143952oc, pp. 661-671, 2016.

[12] Z. Meng, J. Dong, and F. Jiang, "Biatrial ablation versus left atrial ablation with bipolar system in the surgical treatment of atrial fibrillation with mitral valve diseas," Chinese Journal of Thoracic and Cardiovascular Surgery, vol. 30, pp. 482-485, 2014.

[13] M. C. Henn, T. S. Lancaster, J. R. Miller et al., "Late outcomes after the Cox maze IV procedure for atrial fibrillation," The Journal of Thoracic and Cardiovascular Surgery, vol. 150, no. 5, pp. 1168-1176, 1178.e1161-1162, 2015.

[14] H. Liu, L. Chen, Y. Xiao et al., "Early efficacy analysis of biatrial ablation versus left and simplified right atrial ablation for atrial fibrillation treatment in patients with rheumatic heart disease," Heart, Lung and Circulation, vol. 24, no. 8, pp. 789-795, 2015.

[15] J. Gualis, M. Castaño, J. M. Martínez-Comendador et al., "Biatrial vs. isolated left atrial cryoablation for the treatment of long-lasting permanent atrial fibrillation. Midterm recurrence rate," Archivos de Cardiología de México, vol. 86, no. 2, pp. 123129, 2016.

[16] S. H. Downs and N. Black, "The feasibility of creating a checklist for the assessment of the methodological quality both of randomised and non-randomised studies of health care interventions," Journal of Epidemiology and Community Health, vol. 52, no. 6, pp. 377-384, 1998.

[17] J. D. Drews, H. J. Patel, D. M. Williams, N. L. Dasika, and G. M. Deeb, "The impact of acute renal failure on early and late outcomes after thoracic aortic endovascular repair," The Annals of Thoracic Surgery, vol. 97, no. 6, pp. 2027-2033, 2014.

[18] M. Covella, A. Milan, S. Totaro et al., "Echocardiographic aortic root dilatation in hypertensive patients: A systematic reviewand meta-analysis," Journal of Hypertension, vol. 32, no. 10, pp. 1928 1935, 2014.

[19] D. P. Taggart, R. D’Amico, and D. G. Altman, "Effect of arterial revascularisation on survival: A systematic review of studies comparing bilateral and single internal mammary arteries," The Lancet, vol. 358, no. 9285, pp. 870-875, 2001.

[20] J. Arrich, M. Holzer, C. Havel, AM. Warenits, and H. Herkner, "Pre-hospital versus in-hospital initiation of cooling for survival and neuroprotection after out-of-hospital cardiac arrest," The Cochrane Database of Systematic Reviews, vol. 3, Article ID CD010570, 2016.

[21] T. Deneke, K. Khargi, D. Voss et al., "Long-term sinus rhythm stability after intraoperative ablation of permanent atrial fibrillation," Pacing and Clinical Electrophysiology, vol. 32, no. 5, pp. 653-659, 2009.

[22] S. Pecha, F. Hartel, T. Ahmadzade et al., "Event recorder monitoring to compare the efficacy of a left versus biatrial lesion set in patients undergoing concomitant surgical ablation for atrial fibrillation," The Journal of Thoracic and Cardiovascular Surgery, vol. 148, no. 5, pp. 2161-2166, 2014.

[23] J. Wang, X. Meng, H. Li, Y. Cui, J. Han, and C. Xu, "Prospective randomized comparison of left atrial and biatrial radiofrequency ablation in the treatment of atrial fibrillation," European Journal of Cardio-Thoracic Surgery, vol. 35, no. 1, pp. 116-122, 2009.

[24] V. Srivastava, S. Kumar, S. Javali et al., "Efficacy of three different ablative procedures to treat atrial fibrillation in patients with valvular heart disease: a randomised trial," Heart, Lung and Circulation, vol. 17, no. 3, pp. 232-240, 2008. 
[25] L. Calo, F. Lamberti, M. L. Loricchio et al., "Left atrial ablation versus biatrial ablation for persistent and permanent atrial fibrillation. A prospective and randomized study," Journal of the American College of Cardiology, vol. 47, no. 12, pp. 2504-2512, 2006.

[26] F. Onorati, G. Mariscalco, A. S. Rubino et al., "Impact of lesion sets on mid-term results of surgical ablation procedure for atrial fibrillation," Journal of the American College of Cardiology, vol. 57, no. 8, pp. 931-940, 2011.

[27] A. Albage, M. Peterffy, and G. Kallner, "Learning what works in surgical cryoablation of atrial fibrillation: results of different application techniques and benefits of prospective follow-up," Interactive CardioVascular and Thoracic Surgery, vol. 13, no. 5, pp. 480-484, 2011.

[28] P. M. McCarthy, J. Kruse, S. Shalli et al., "Where does atrial fibrillation surgery fail? Implications for increasing effectiveness of ablation," The Journal of Thoracic and Cardiovascular Surgery, vol. 139, no. 4, pp. 860-867, 2010.

[29] M. Guden, B. Akpinar, B. Caynak et al., "Left versus biatrial intraoperative saline-irrigated radiofrequency modified maze procedure for atrial fibrillation," Cardiac Electrophysiology Review, vol. 7, no. 3, pp. 252-258, 2003.

[30] L. K. Soni, S. R. Cedola, J. Cogan et al., "Right atrial lesions do not improve the efficacy of a complete left atrial lesion set in the surgical treatment of atrial fibrillation, but they do increase procedural morbidity," The Journal of Thoracic and Cardiovascular Surgery, vol. 145, no. 2, pp. 356-363, 2013.

[31] J. B. Kim, J. H. Bang, S. H. Jung, S. J. Choo, C. H. Chung, and J. W. Lee, "Left atrial ablation versus biatrial ablation in the surgical treatment of atrial fibrillation," The Annals of Thoracic Surgery, vol. 92, no. 4, pp. 1397-1405, 2011.

[32] J. R. Breda, A. S. C. R. Breda, R. G. Ragognette et al., "Comparison of uniatrial and biatrial radiofrequency ablation procedures in atrial fibrillation: Initial results," Heart Surgery Forum, vol. 14, no. 5, pp. E271-E275, 2011.

[33] G. S. C. Geuzebroek, P. K. E. W. Ballaux, N. M. Van Hemel, J. C. Kelder, and J. J. A. M. T. Defauw, "Medium-term outcome of different surgical methods to cure atrial fibrillation: Is less worse?" Interactive CardioVascular and Thoracic Surgery, vol. 7, no. 2, pp. 201-205, 2008.

[34] T. Deneke, K. Khargi, B. Lemke et al., "Intra-operative cooledtip radiofrequency linear atrial ablation to treat permanent atrial fibrillation," European Heart Journal, vol. 28, no. 23, pp. 2909-2914, 2007.

[35] W. H. Ryan, H. B. Prince, G. H. Wheatley et al., "Experience with various surgical options for the treatment of atrial fibrillation," Heart Surgery Forum, vol. 7, no. 4, pp. E333-E336, 2004.

[36] Y. Takami, K. Yasuura, Y. Takagi et al., "Partial maze procedure is effective treatment for chronic atrial fibrillation associated with valve disease," Journal of Cardiac Surgery, vol. 14, no. 2, pp. 103-108, 1999.

[37] S. Lazar, S. Dixit, F. E. Marchlinski, D. J. Callans, and E. P. Gerstenfeld, "Presence of left-to-right atrial frequency gradient in paroxysmal but not persistent atrial fibrillation in humans," Circulation, vol. 110, no. 20, pp. 3181-3186, 2004.

[38] F. Gaita, L. Calò, R. Riccardi et al., "Different patterns of atrial activation idiopathic atrial fibrillation: Simultaneous multisite atrial mapping in patients with paroxysmal and chronic atrial fibrillation," Journal of the American College of Cardiology, vol. 37, no. 2, pp. 534-541, 2001.

[39] P. Sanders, O. Berenfeld, M. Hocini et al., "Spectral analysis identifies sites of high-frequency activity maintaining atrial fibrillation in humans," Circulation, vol. 112, no. 6, pp. 789-797, 2005 . 


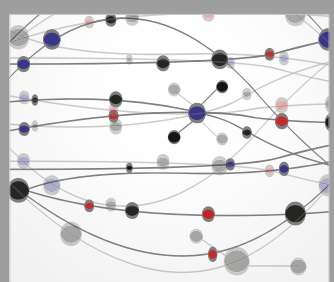

The Scientific World Journal
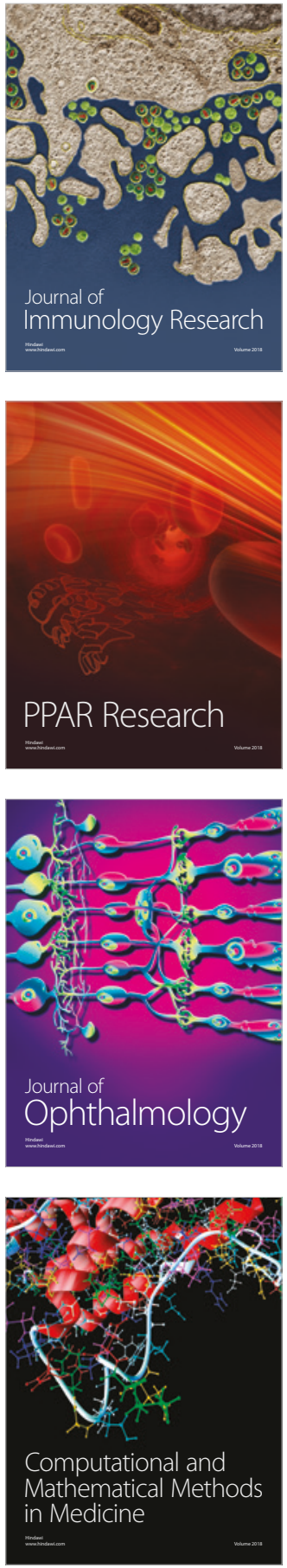

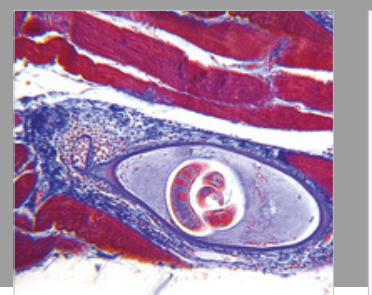

Gastroenterology Research and Practice

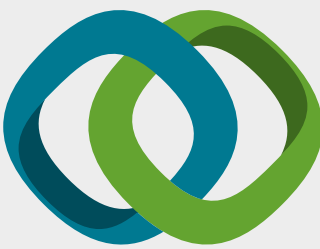

\section{Hindawi}

Submit your manuscripts at

www.hindawi.com
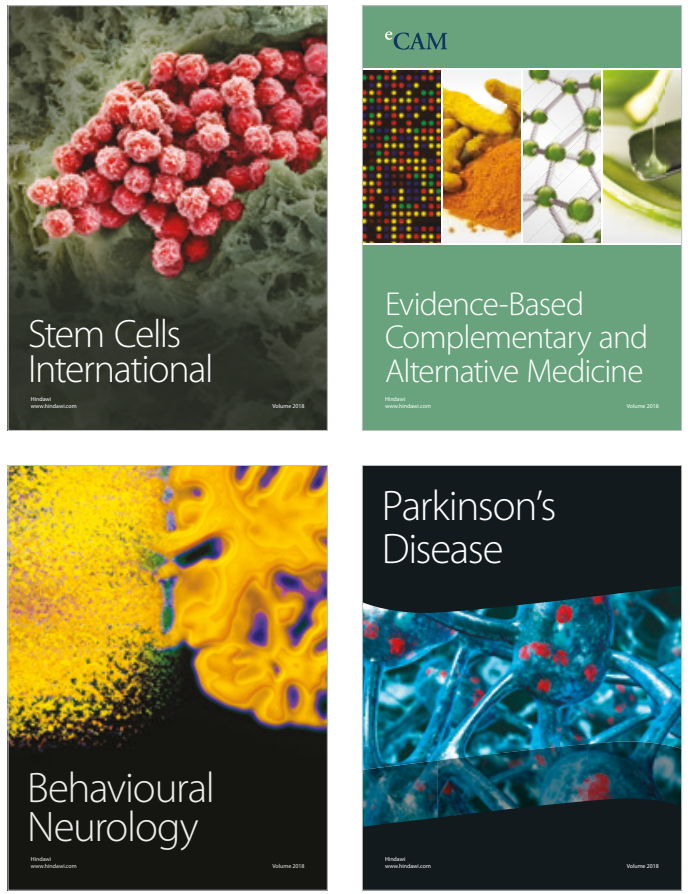

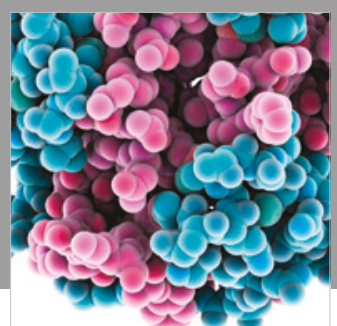

ournal of

Diabetes Research

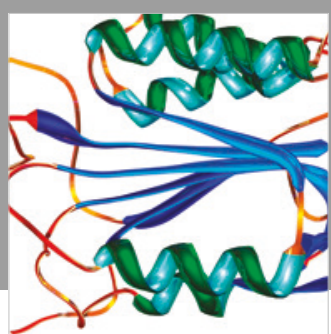

Disease Markers
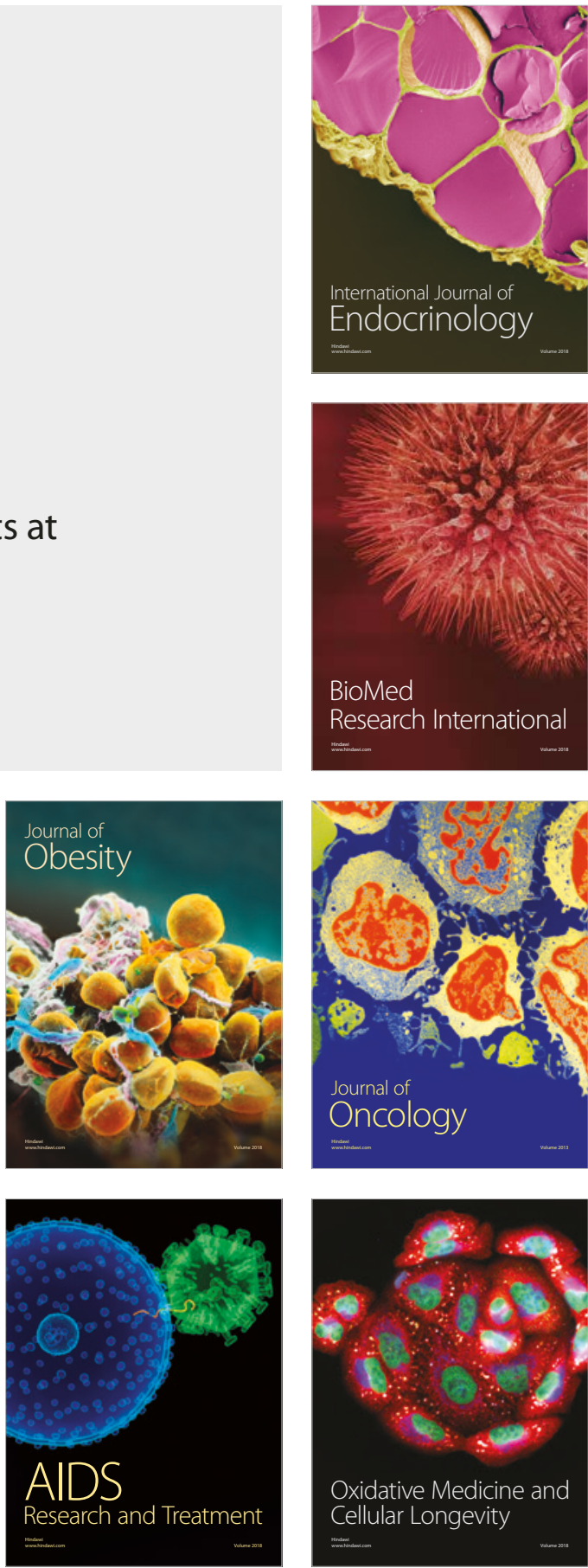